\title{
Conservative periodontal surgery for treatment of intrabony defects is associated with improvements in clinical parameters
}

\author{
Abstracted from \\ Graziani F, Gennai S, Cei S et al. \\ Clinical performance of access flap surgery in the treatment of the intrabony defect. \\ A systematic review and meta-analysis of randomized clinical trials. J Clin Periodontol 2012; 39: 145-156. \\ Address for correspondence: Filippo Graziani, Department of Surgery, \\ Unit of Dentistry and Oral Surgery, University of Pisa, Pisa, Italy. E-mail: filippo.graziani@med.unipi.it
}

\section{Question: What is the rate of tooth survival and clinical attachment level gain, probing pocket depth reduction and gingival recession increase, of teeth affected by periodontitis related intra-bony defect, 12 months after conservative periodontal surgery?}

Data sources The Cochrane Oral Health Group specialist trials, Medline and Embase databases were searched. The Journal of Clinical Periodontal Research and the Journal of Clinical Periodontology were hand searched.

Study selection Randomised controlled trials involving an intervention of surgical flap or periodontal pocket surgery including simplified, modified, access, Widman, or papilla preservation and disease or intrabony/intraosseous defect were included. Two authors screened and selected the studies.

Data extraction and synthesis Data extraction and risk of bias assessment were conducted by two authors. Primary outcomes were tooth survival, clinical attachment gain, probing depth reduction and gingival recession increase. Weighted means and forest plots were calculated and meta-analysis performed.

Results Thirty-one studies were included for data analysis. Twelve months after conservative surgery, tooth survival was $98 \%$, clinical attachment loss gain $1.65 \mathrm{~mm}$ (95\% Cl 1.37-1.94), probing depth reduction $2.8 \mathrm{~mm}(\mathrm{Cl} 2.43-3.18)$ and gingival recession increase 1.26 $\mathrm{mm}$ (Cl 0.94-1.49). Longer follow up showed similar results. There was insignificant evidence to suggest that clinical bone increased with this surgery.

Conclusions The treatment of intrabony defect with conservative surgery is associated with high tooth retention and improvement of periodontal clinical parameters. Clinical performance may vary according to the type of surgical flap used.

\section{Commentary}

The risk of periodontitis increases for patients who have periodontal intraosseous lesions. ${ }^{1}$ One way of re-establishing periodontal health for people with intraosseous lesion associated periodontitis is to re-establish proper plaque control ${ }^{2}$ using a surgical procedure. Although treating these lesions by regenerative procedures is superior, there are still significant benefits of treating intrabony defects using conventional conservative surgery. ${ }^{3}$ As Cortellini suggests, ${ }^{4}$ regenerative surgery may not always be the first choice for this type of periodontitis, as conservative surgery has a more favourable cost-benefit outcome. Graziani's systematic review is helpful for healthcare providers who provide treatment for such patients as the paper reviews high quality randomised controlled trials that investigate the performance of conservative surgery.

While three databases were searched and no language limits were applied, additional databases eg LILACS or the Chinese biomedical database could have identified more relevant non-English studies. In is also unclear as to what time period the journals of Periodontal Research and Clinical Periodontology were searched, or if any trial registers were searched.

The authors provide a useful summary of the characteristics of the studies that were chosen as part of the systematic review including for each study; methods, interventions, outcomes, tooth-loss, site and funding. Like most oral diseases there is a social gradient between household income and periodontitis, as well as a link between the disease and age. ${ }^{5}$ To determine the relevance of each study to one's individual practice population it is important to understand, if possible, these variables. It would have been beneficial to include a little more information about the types of participants within the study including smoking information (on participants in those studies where this was provided), the age and if possible the socio-demographic information about the participants. The authors note the type of practice where the surgery was performed; however private practice does not necessarily give an indicator of socio-demographic information. A private practice in a country without comprehensive state funded dentistry programmes (eg Australia) can be quite different from a private practice in the United Kingdom.

As recommended by the Cochrane Collaboration the authors used a detailed search protocol based on PRISMA, (www.prismastatement.org) with two reviewers assessing the trials for inclusion into the review. The authors also took the additional step of being calibrated against a third author. 


\section{PERIODONTOLOGY}

The authors should be congratulated for their thoroughness when it comes to assessing quality. The authors of the review independently used Cochrane criteria to assess quality, and contacted authors if they were unsure about the quality. They excluded articles in the final selection if the authors could not provide sufficient detail about their individual studies. The quality evaluation included an analysis of allocation concealment, blinding of personnel and outcome assessors. Articles assessed as poor quality were not included within the analysis. The authors argue for high-quality studies with long term follow-up to better understand the performance of conservative surgery in this situation.

This paper provides a very useful summary of current information regarding the sequelae to conservative periodontal surgery, although the use of abbreviations and acronyms make it less accessible to the non-specialist reader. There is a number of forest plots presented in this paper. When looking at each of the individual studies on clinical attachment gain these forest plots illustrate the wide treatment effects within the different studies, with a number of studies having a $95 \%$ confidence interval where the treatment effect was either zero or included either loss of clinical attachment or gain.

This is where meta-analysis has its advantage. By 'pooling together' a number of studies with similar populations a metaanalysis can provide a more accurate indication of the overall effect of a procedure. In this illustration the pooling of the results gave not only an estimate of potential treatment effect, but a narrow margin of that effect. When the results from these studies are pooled via meta-analysis, conservative surgery lead to a 12-month clinical attachment gain of $1.65 \mathrm{~mm}$ (with a 95\% CI of 1.36-1.97, meaning we can be $95 \%$ certain that the clinical attachment gain lies between 1.36 and $1.97 \mathrm{~mm}$.) The clinical attachment after 12 months was $1.97 \mathrm{~mm}$ (95\% CI 1.13-2.81). This is a useful result for clinicians in practice, highlighting the positive effects of conservative surgery.

\section{Practice point}

- Conventional periodontal surgery reduces probing pocket depth reduction, and gingival recession.

\section{Brett Duane}

Department of Public Health, NHS Fife, Cameron Hospital, Windygates, UK.

1. Papapanou PN, Tonetti MS. Diagnosis and epidemiology of periodontal osseous lesions. Periodontol 2000; 22: 8-21.

2. Rosling B, Nyman S, Lindhe J. The effect of systematic plaque control on bone regeneration in infrabony pockets. J Clin Periodontol 1976; 3: 38-53.

3. Needleman I, Tucker R, Giedrys-Leeper E, Worthington H. Guided tissue regeneration for periodontal intrabony defects--a Cochrane Systematic Review. Periodontol 2000; 37: 106-123

4. Cortellini P, Tonetti MS. Focus on intrabony defects: guided tissue regeneration. Periodontol 2000; 22: 104-132.

5. AlHW 2010. Socioeconomic variation in periodontitis among Australian adults 2004 2006. DSRU report no. 50. Cat. No. DEN 207. Canberra: AlHW. Viewed 2 November 2012 http://www.aihw.gov.au/publication-details/?id=6442472416

Evidence-Based Dentistry (2012) 13, 115-116. doi:10.1038/sj.ebd.6400898 